cord. It is quite possible that it may be; and if so, we may expect to find a further gradual extension of the disease, with increase and alteration of the symptoms, true paralysis becoming ultimately developed.

Meanwhile it is clear that the object to be kept in view in treatment is the diminution of the excitalility of the spinal cord. The application of icebags along the spine appears to be invariably followed by temporary improvement. When they have been applied for some time, the patient bends bis legs quite readily; but when he attempts to walk, little difference is noticed. Ergot of rye has failed to do good; conium has also failed; chloral hydrate appeared to prove in some degree beneficial, but latterly has been found to be of little use. Other remedies must be tried, in the hope that the irritability may be overcome.

Charlotte-square, Edinburgh.

\section{A CASE OF FEMORAL ANEURISM.} By Fleet-Surgeon JohN ElliotT, R.N.

(Communicated by the Dromctor-General of thi Medical Department OF THE NAVY.)

S. T_, gnnner, Royal Marine Artillery, aged thirtyseven, single, of spare habit, thin, sallow features, and prematurely-aged appearance, was admitted into the Naval Sick Quarters at Halifax on the 6th of June, 1874. During eighteen years' service he has been in the hospital with intermittent fever, pneumonia, syphilis (not constitutional), and bubo. Otherwise he has enjoyed good health, occasionally, however, complaining fir days at a time of a stitch beneath the left nipple. He is of temperate habits, and does not smoke. For the last two years and a half he has been employed as coachman to an officer on this station. The patient stated that on Saturday, the 30th of May, he had a number of heary packages to lift and place in position, and among them a barrel of vegetables weighing $3 \mathrm{cwt}$. The following day, though not complaining, he did not go out, and on the Monday "felt curious," finding a great stiffness from the knee to the hip when he got up. These symptoms increased during the day, and prevented his sleeping at night. He felt "as if he had been beaten about the thigh with a stick." On Tuesday morning, when getting out of bed, he noticed a lump, the size of a " hen's egg," in his left groin, beating forcibly. He went about his customary duties, however-washing a carriage, cleaning harness, \&c. Towards the evening the limb felt numb, and he applied a turpentine stupe. The following day the numbness had disappeared, though the tumour had increased in size, and the pulsation was stronger. Went about his duties as usual-driving, \&c.; and at 11.30 r.M., on his way home, he felt the cold very much. On Thursday there was a continuance of the same signs. On Friday he took to bed; and on Saturday morning, the 6 th of June, at 9 A.M., he applied for relief at the Naval Sick Quarters, and was at once admitted.

Present condition. - Upon examination a large tumour, measuring 5 in. (transversely) by 4 in. (vertically), somewhat globular in shape, but compressed on its surface, and pulsating visibly with great force, was found at the upper and anterior part of left thigh, occupying the greater part of Scarpa's space and whole of inguinal region, pushing up Poupart's ligament, and obscuring situation of anterior superior iliac spine externally. Upon palpation it was found distinctly circumscribed, hard, and tense, with greatly thickened coats, and distensile pulsation. Its surface was marked by a cicatrix two inches in length, where a bubo had been opened some time before. A soft bruit was heard all over the tumour, and with greatest distinctness internally over the artery. The heart sounds were found normal, though somewhat deficient in strength, and the general health was good.

General record.-Patient was at once placed in bed, and ordered a mixture containing two scruples of tincture of digitalis and one grain of tartar emetic, one-eighth part three times daily; low diet with milk. Digital compression was applied for two hours over external iliac artery, in close vicinity of tumour, and had the effect of almost stopping the pulsation, the bruit disappearing; but the size of the tumuur was not lessened, nor could it by pressure be emptied of its contents. Patient complained greatly of numbness of thigh below seat of aneurism during and after its application, and the temperature of the leg and thigh of the affected side was found lower than that of the other. The artery was again compressed for an hour at 2.30 P.M., but had to be stopped on account of the pain to which it gave rise. It was again attempted at 4 P.M., but could not be borne. The pulsation in tumour is stronger -85 . Ice was applied at 6 P M., and at 8 P.Mr. it had lessened somewhat.

June 7 th.-Digital compression was tried, but could not be borne. Slept three hours last night. Complains of numbness of left leg, and hot-water tins were placed around it. Measurement round left thigh over tumour, 22 inches. At 8 P.M. complained of numbness of limb. Stop ice. Temperature in flexure of left knee, $97 \cdot 4^{\circ} \mathrm{F}$.; right ditto, $96^{\circ}$. Ordered one ounce of castor oil, as bowels were confined.

8th.-Tumour measures 6 in. (transversely) by 4 in. (vertically); left thigh around tumour, $23 \frac{1}{\mathrm{~s}}$ in.; measurement round upper right thigh, 20 in. There has been an increase of one inch in a transverse direction. No numbness in thigh ; slept well; bowels have acted freely. Temperature in left flexure of knee, $952^{\circ}$; right ditto, $954^{\circ}$. Ordered the following mixture:-Tannic acid, two drachms; tincture of digitalis, half a drachm; to eight ounces of water; one ounce every hour. Pulsation in tumour, strong distensile, 89. The thigh was flexed on the abdomen, and bandaged in that position for about four hours, when the limb was straightened, patient complaining of the constrained position. At 4.5 limb again flexed on abdomen, till 8.30, when numbness and pain shooting down inside of thigh, with heat of parts coming on, it was stopped. Temperature of right flexure of knee, $97 \cdot 2^{\circ}$; left ditto, $98 \cdot 1^{\circ}$. Bowels moved twice to-day.

9 th.-Slept well; pulse 80 ; tumour measures $3 \frac{1}{4}$ in. vertically, 6 in. transversely ; temperature of right flexure of knee, $963^{\circ}$, left ditto, $973^{\circ}$; pulsation quieter; complains of numbness down the centre of the thigh; temperature near tumour, $98^{\circ}$. By means of a tourniquet kept in position by relays of nurses, pressure was kept up for three hours and $a$ half, the circulation through tumour being lessened considerably. It was again applied from 9 till 10 P.M.

10th.-Slept from 9 till 2 A.M. Temperature of right leg $98 \cdot 1^{\circ}$; of left, $96.4^{\circ}$. Tumour measures $6 \frac{1}{8}$ in. transversely $4 \frac{1}{4}$ in. vertically. Measurement around thigh 26 in. Pulsation in tumour 90. Pulsation can be felt in both tibials to-day for the first time; there is a slight difference in the pulsations. Surface of skin over tumour slightly discoloured and finely rugated. Bruit heard over whole surface. Tumour more compressible than it was. At intervals pressure was kept up, as during yesterday. At 4.15 Carte's tourniquet was placed in position and the pad screwed down till pulsation or bruit could not be detected in the tumour. It had to be removed on account of faintness and palpitation of heart, which attacked the patient shortly after its application. Great distress continued for the rest of the day. A morphia draught was given at night.

11 tb.-Has passed a good night. The tumour has increased in size, measuring $6 \frac{1}{2}$ in. transversely and $4 \frac{1}{2}$ in. vertically. The skin covering it is livid and discoloured, and corrugated. To the touch it is softer at its most prominent part. The pulsation has increased in strength. Temperature of right flexure of knee, $964^{\circ}$; of left ditto, $981^{\circ}$. Pulsation apparent in posterior tibial artery of right side. Bruit loud all over the tumour; most distinct internally. At 9 A.M. patient was given a dose of castor oil. At $100^{\prime} \mathrm{clock}$, as the bowels had not acted, a simple enema was administered, a small quantity of fæces coming away.

A consultation was held with the medical officers of the ship, Dr. Jennings of the Halifax Civil Hospital, and Dr. Douglas, Army Medical Staff; and it was decided to tie the external iliac, or, if impracticable, the common iliac artery.

Operation.-Chloroform was administered, and at 12.15, patient being well under its influence, I made an incision five inches in length, extending from a little inside and two fingers' breadth above Poupart's ligament, at its centre, upwards and outwards, to within three fingers' breadth of 
the crest of the ilium; and the skin, superficial fascia, external oblique, internal oblique, and transversalis muscles having been divided, the transversalis fascia was exposed, and carefully divided on a director to the full extent of the external wound; the peritoneum was then loosened from the surrounding cellular tissue and drawn up towards the mesial line of the abdomen, and the sheath of the vessels exposed. This having been worked through gently, the ligature (a twisted hempen one) was passed from within outwards, and tied, pulsation at once ceasing in the sac and in the vessel beneath it. Four small vessels required ligature during the operation. The parts through which the incisions were made were found greatly congested from pressure, but the artery appeared to be healthy. The wound was sponged with carbolic-acid lotion, and its edges brought together by seven points of interrupted suture (silver wire); carbolic dressing was then applied with a slight compress and bandage. The whole of the left limb was then enveloped in cotton wool with a flannel bandage and surrounded by warm-water tins. Pulse 92. No change in tumour. At 1.30 one drachm of solution of morphia was given, and again at 3. At 4 o'clock he complained of numbness of the left leg. Pulse 94. Slept for two hours towards evening. He was ordered one grain of opium pill every fourth hour. Pulse 100. At 10 P.M. comfortable; no pain; leg warm; pulse 100; passed urine. At 11 P.M. was sleeping.

June 12th. 9.30 A.M. : Passed a good night; pulse 95; temperature in axilla $101.4^{\circ}$; thirsty; restless. Abdomen distended and tympanitic. Slight oozing from wound, staining bandages. Complains of numbness of left foot; and on examination this is found to be very cold. Tins of hot water renewed. To continue the opium pill. Has taken nothing but a little milk-and-water since the operation.12 A.M.: Sleeping. - 1 P.M.: Has passed water; no pain; pulse full, 102.-5 P.M.: Pulse 124; temperature 101.4:; respiration 24 . Temperature of left leg (bot-water bottles in vicinity) $964^{\circ}$. - 6 P.M. : Pulse 130, small, soft, and compressible ; temperature $1024^{\circ}$; restless ; tongue white, parched; great thirst; eyes bright, suffused; skin dry and nough. When seen at night he was perspiring a little. Had passed urine involuntarily at times in bed during the day; but before visit had passed a vesselful without diffculty. Complains greatly of flatulence.

13th.-Did not sleep last night. Pulse 124, soft, small, compressible; tongue white and parched ; restless, throwing off clothes. Suffers greatly from tympanites. Temperature in axilla $992^{\circ}$; in vicinity of tumour $1001^{\circ}$. Tumour measures 52 in. transversely, 34 in. vertically, and feels harder. No evidence of returning pulsation. Temperature of left leg $95 \cdot 3^{\circ}$; of right do. $94,3^{\circ}$. Was ordered ice to relieve thirst, and beef-tea during the day. Abdomen dis. tended, tympanitic, and painful on pressure. Not much discharge from wound. Tumour hard and tense. Left leg and foot warm. Has been taking opium pills during the day, but has only slept two hours since 11 o'clock last night. Ordered one drachm of solution of muriate of morphia; to be repeated if he does not sleep.

14th. -7 A M. : Slept for two hours last night, the rest of the time dozing. Pulse 135; skin warm; left leg and toes warm. Upon removing the dressings, which were slightly stained with discharges, the wound was found looking healthy, and united for part of its extent. At its lower angle, where the ligature came through, there was a slight sanious discharge. The wound was redressed, and soiled linen removed. The abdomen continnes distended and tympanitic. Got nourishment in small quantities at repeated intervals during the day, and continued taking the opium pills. Ice was also given at intervals. The bowels have not acted since the operation. The wound discharged more freely after redressing. At 9 P.M. the patient was found very restless; the pulse 142 ; tongue thickly furred; vomited a little during the afternoon; is slightly delirious. 21 P.M. : Sleeping.

15th.-Awoke at 8 A.M. covered with a cold clammy sweat; extremities cold; pulse 72, irregular; countenance anxious; passed urine in bed; conscious. Stimulants were at once administered, and hot-water tins renewed around extremities.-10 A.M.: Had rallied a little. Was given brandy, egg-mixture, and beef-tea at short intervals. A large piece of spongio-piline, wrung out of hot water, was kept constantly applied to the abdomen. Passed about a pint of dark-coloured turbid urine. At 12 A.M. his pulse was 150, small, soft, and compressible. Pain on pressure over the abdomen. Wandering slightly; perspiring. At 1.30 there was nausea and slight vomiting. Brandy was administered, and a draught containing half a grain of muriate of morphia.-4 P.M. : Slept a little since last visit. Gradually sinking. A marked change for the worse since last visit. Pulse rapid, fluttering; cannot be counted; talking incoherently; floccitatio. -5 P.M. : Pulse 199, fluttering; cold clammy sweat over the body. Floccitatio continues. Sighing respiration.-7.15: Sinking rapidly; rapid fluttering pulse. Stimulants continued at short intervals. Unconscious.-8.45: Expired.

Autopsy (performed twenty-four hours after death).Rigor mortis absent; body emaciated; abdomen distended and tympanitic; scrotum dark and congested. A tumour, soft on palpation, $5 \cdot 2$ in. transversely, $3 \frac{1}{2}$ in. vertically, is situated in left groin and Scarpa's space. About 2 in. above it is a wound $5 \frac{1}{2}$ in. in length, extending outwards from the centre of Poupart's ligament, and almost united in the intervals between seven points of silver suture. Lungs healthy; old adhesions connecting lungs and pleura on both sides. About two ounces of clear yellow serum in cavity of pericardium. Heart small; flaccid; more than the normal deposit of fat in interventricular furrows; slight warty vegetations on anterior segment of mitral valve; slight roughening on anterior surface of ascending aorta; decolorised coagulum in right auricle; dark clot in pulmonary artery. The intestines were found distended with gas; a portion of the left lower edge of the omentum, four inches in extent, was congested, adherent to in testine beneath; lymph was effused on its surface. Liver, spleen, and kidneys bealthy. A piece of the lower part of the ileum, six inches long, was intensely congested; the cæcum below it contained an accumulation of hardened fæces; about four inches of the colon at the sigmoid flexure was also found congested, the rectum below containing some hardened fæces. Gall bladder distended with inle, dark externally. The intestines were removed; the surgical wound in abdominal walls was found united superficially almost throughout the whole extent. More deeply, however, such was not the case, the muscles, \&c., in the line of incision being congested and disorganised. Pus, green in colour, extended upwards for some dirtance on abdominal walls, between planes of muscles. Upon raising the peritoneal sac from the iliac fossa, diffuse cellular inflammation was found extending upwards as far as left kidney. The pus in the iliac tossa was of a pink tinge. The surgical wound was two inches above and parallel to Poupart's ligament. Upon removing the integument and superficial fascia from the front of the left thigh, the aneurismal tumour, tightly bound down by the sartorius muscle and the fascia lata, was discovered. Upon cutting across the former the tumour assumed a more conical shape. Its most prominent part was found greatly thinned, the same condition existing externally. It was with difficulty dissected out of its bed in the psoas, iliacus, and pectineus muscles, a considerable portion of these, together with a part of the capsular ligament of the hip-joint, having to be removed on account of their connexion with the tumour. Som $\theta$ doubt existed as to whether a depression observed on the head of the femur, below the acetabulum, was not due to pressure of the tumour, but on account of the history of the short duration of the disease, it was decided not. A few nerves were found stretched across its surface. The aneurism was formed on the external side of the femoral artery, from which it extended outwards, probably due to rupture of its internal and middle coats-its external coat, together with the sheath of the vessels, which was found greatly thickened, forming the coverings. The measurements were $3 \frac{1}{2}$ in. vertically and horizontally, 9 in. round greatest circumference. Its contents were fluid, a considerable portion of them (dark blood) having escaped by accidental rupture. The ligature was found firmly applied on the external iliac artery, two inches above the tumour, and two inches below the origin of the internal iliac artery. The femoral artery was involved for an inch and a balf in the formation of the tumour; it appeared healthy. The femoral vein was found adherent, especially at the centre; above this its coats were thinned and dilated; below, greatly thickened (six lines). A quarter of an inch below the emergence of the femoral artery from the tumour two arteries were given off-the profunda femoris, hard and nodulated to the touch, and the exter! al circumflex ilii. 
The walls of the tumour were excessively thinned throughout, more particularly above and externally. The muscles in immediate contact were softened and disorganised.

Remarks. - The cause of the fatal termination in this case was diffuse cellular inflammation. Owing to the large size of the tumour, the parts around were subjected to considerable pressure, causing congestion \&c., and were unable to regain their vitality after the operation.

\section{NOTE ON}

THE TREATMENT OF SCARLET FEVER BY THE EXTERNAL APPLICATION OF COLD WATER.

WITH TWO CASES.

BY JOHN EUWIN EDDISON, M.D.,

PHYNYOIAY TO THR LERDS INFIBMABY, THB FEVER HOSPITAL, HTC.

(The Cases reported by Dr. STEzL, Resident Medical Officer at the Fever Hospital.)

(Concluded from page 341.)

CASE 1.-S. F-, aged eight, was admitted into the Leeds Fever Hospital on the morning of the 13th of May, 1875, being the third day of illness. On admission, the following notes were made:- "Face congested and of a dusky hue. A nearly uniform blush of redness is observable over the upper part of the chest anteriorly, and upon the hands there is a faint but characteristic scarlatinal rash; elsewhere there is no rash. Temperature in the axilla $1042^{\circ} \mathrm{F}$. The skin is dry, and feels extremely hot; tongue thickly furred, red at the tip and margin; fauces and tonsils much congested and swollen; diffuse, partly codematous, swelling at the angles of the jaw, accompanied by pain and tenderness. Over the lungs, posteriorly and inferiorly, a few coarse bronchitic rhonchi; elsewhere a vesicular murmur. Respiration 24 per minute; action of heart excited; pulse 144; heart-sounds clear. Patient is very drowsy, and her breathing heavy. Urine contains no albumen; sp. gr. 1020." She was ordered a tepid pack, two sheets, each applied for ten minutes. When taken out the temperature had fallen to $101 \cdot 8^{\circ}$. This was at noon. At 1 P.M. it had again risen, and reached 105. $3^{\circ}$. Another pack was immediately ordered, cold instead of tepid water being used. After thirty minutes, three sheets having been applied one after another, her temperature had again fallen, this time to $100^{\circ}$. A fourth and fifth sheet were applied however, but, on her being placed in the latter, slight shivering with chattering of the teeth supervened; when taken out and dried the temperature was found to have risen to $102^{\circ}$. The patient was now slightly delirious, and still very drowsy. At 5 P.M. the temperature was $105^{\circ}$; pulse 144, and weak. Powerful grinding movements of the lower jaw have supervened. She was now placed in a tepid bath, the water of which was gradually cooled down. During the bath she appeared mnre sensible, but the grinding movements of the jaw continued. Temperature, when taken out, $99^{\circ}$. She was kept in thirty minutes. The pulse was scarcely perceptible ; but this seemed due chiefly to a local contraction of the arterial coats, as the action of the heart was ascertained by palpation and auscultation to be still vigorous. At 7 P.M. the temperature was $1016^{\circ}$; at 7.30 , $103^{\circ}$; at $8,1032^{\circ}$. A patch of deep-coloured rash was now observable on and above the elbows. At 10 P.M. the rash was well out on the arms and forearms. It was of a deep colour, and coarsely mottled in aspect. The patient was very drowsy, and slept with the eyelids open. Pupils normal ; temperature $105^{\circ}$. At 11 P.M. she was again placed in a tepid bath, which was cooled down as before. During this bath the rash upon the arms became intense. The patient talked rationally while immersed, but occasionally rambled. Grinding movements of the jaw reappeared. She was kept immersed for forty-five minutes, and when taken out was much less drows, while her temperature had fallen to $956^{\circ}$. Half an hour aster the bath it had risen one degree. The treatment adopted, besides bathing, consisted in the application of cold compresses to the throat, $3 \mathrm{oz}$. of wine in the twenty-four hours, and nourishment in the form of strong beef-tea and milk. The throat was also sponged with Condy's fluid.

May 14th (fourth day of disease). - Temperature during the night: 1 A.M., $101^{\circ} ; 2,103 \cdot 2^{\circ} ; 3,104.2^{\circ} ; 4,104.2^{\circ}$; $5,1046^{\circ} ; 6,1034^{\circ} ; 7,104: 2^{\circ} ; 8,1038^{\circ} ; 9,1036^{\circ} ; 10,1046^{\circ}$. Pulse 148. After last night's bath she slept quietly for three hours, and then woke up asking for a drink. This morning she continues very drowsy, her breathing heavy and snoring. Rash well out on the arms, but not so bril. liant as during the bath. Taking nourishment well. Tongue red and characteristic of the disease. Passing urine in bed. Bowels open. - 2.30 P M. : Rash brilliant on arms; well out on legs and thighs. Temperature has stood persistently at a few points above $104^{\circ} .-4.30$ P.M. : Temperature $104.4^{\circ}$. Placed in the bath again. The temperature in ten minutes had fallen to $101^{\circ}$, in twenty minutes to $996^{\circ}$, and in thirty. five minutes to $955^{\circ}$, when she was taken out. - At $5.30 \mathrm{P}$. the temperature was $96^{\circ}$, and at $6.30,101 \cdot 6^{\circ}$. 7 P.M. : Pulse 136; sleeping quietly. During the last bath she was quite conscious and talked a good deal.-10 30 P.Mr.: Rash intense on arms ; over the trunk a distinct mottling. Temperature $1044^{\circ}$. Placed in bath; kept in fifty minutes. Temperature when taken out $962^{\circ}$.

15th (fifth day of disease). - Temperature during the night: at 1245 A.M., $101.4^{\circ} ; 1.30,1022^{\circ} ; 2.30,104^{\circ}$. At 230 she was placed in a wet pack, and the temperature at $3 \mathrm{~A} . \mathrm{M}$. had fallen to $101^{\circ}$.* At 4 A.M. it had risen to $103.4^{\circ}$ 。 At 6 she was again placed in a pack, the temperature at 6.30 baving again fallen to $101^{\circ}$. Pack continued, sheets changed every ten minutes, and cold water used. At 7 the temperature was $102 \cdot 2^{\circ}$; and at 8 it was $102 \cdot 8^{\circ}$, in spite of pack. She was then taken out. Temperature at 9 A.M., $1032^{\circ}$; at $10,1038^{\circ}$. Rash universal; intense on extremities, resembling erysipelas. Tongue red, and as yesterday. Bowels open twice. Pulse 14.4. Patient is more intelligent and much less drowsy. At 11 A.M. the temperature was $104^{\circ}$, and at 12 noon $105^{\circ}$. The patient was again ordered a cold wet pack, which proved unsuccessful at first; but by placing her, wrapped in a sheet, in an empty bath, and sponging her with cold water, the temperature was at last reduced to $984^{\circ}$ (at 2 P.M.) At 3 P.M. the temperature was $984^{\circ}$ and the pulse 128, the beart-sounds being clear and its action less tumultuous. A.t 3.30 the temperature was $99^{\circ}$, at 4.30 it was $1024^{\circ}$, and at 5.45 P.M. $103.4^{\circ}$. In ten minutes after being placed in a bath the temperature fell to $98^{\circ}$; she was kept in, however, for five-and-twenty minutes. At 7.30 the temperature had risen to $1004^{\circ}$, and at 10.30 to $1036^{\circ}$. At 10.45 the patient was delirious, talking of school, \&c. Temperature 104. Drowsy again. Pulse 144. Breathing heavy; respiration 26 per minute. The patient was placed in a bath, with the water at $90^{\circ}$ cooled down to $68^{\circ}$. She was kept in forty-three minutes. During the bath the pulse was 128 and the respiration 26 . Mind quite clear after the bath. The thermometer could not be got to register bigher than $95^{\circ}$ in the axilla.

16th.-Temperature at 1 A. . $99 \cdot 8^{\circ}$; at $4,103 \cdot 2^{\circ}$. On being placed in a batb, the temperature was reduced in fifteen minutes to $101.4^{\circ}$, in thirty minutes to $964^{\circ}$, and in forty five minutes to $962^{\circ}$, when she was taken out. At 6 A.M. the temperature was $98^{\circ}$; at $10,1026^{\circ}$. Was sick in the last bath. Patient usually drinks freely of milk or beef-tea before entering and during the bath. Respiration 28 per minute; pulse 144; tongue dry and glazed; bowels not moved; passes urine in bed; takes food pretty well; breath fetid. At 12.30 P.M. the temperature was 103.4. In ten minutes after being placed in the bath the temperature had fallen to $998^{\circ}$, and in thirty minutes to $95.2^{\circ}$, when she was taken out. At 5.20 P.M. the temperature was $103^{\circ}$. She was placed in the bath at 6 P M., and in ten minutes the temperature had fallen to $98.4^{\circ}$, and in thirty minutes to $952^{\circ}$, when she was taken out. At 7 P.M. the pulse was 96 (taken with the stethoscope also). The temperature at 10.15 was $101 \cdot 8^{\circ}$.

17 th (seventh day of disease).-Temperature at midnight, $102^{\circ}$; 1 A.M., $102 \cdot 6^{\circ} ; 2,1022^{\circ} ; 3$, ditto; $4,102^{\circ} ; 5,1022^{\circ}$. 6 , ditto; $7,101 \cdot 8^{\circ} ; 8,102^{\circ} ; 9,101.4^{\circ} ; 10,101^{\circ}$. Tongue moist, but clammy; bowels moved thrice; breath very fetid; fauces still congested; tonsils enlarged, white pultaceous 